\title{
THE SHELBY CULLOM DAVIS CENTER FOR HISTORICAL STUDIES
}

\section{Popular Culture}

In I975-76 the Shelby Cullom Davis Center for Historical Studies will continue its Research Seminar on Popular Culture. The Seminar will examine all aspects of popular culture, such as working class culture: the popular press; the ideological background to popular movements of protest and rebellion; popular organizations such as youth groups and secret societies; popular activities such as festivals and games; the means of propaganda and social control used by those in authority to influence and mold public opinion; and the relation between popular and high culture. The Seminar plans to gather together experts in different periods and countries, and hopes to attract scholars from other disciplines than history.

The Center will offer a limited number of Research Fellowships for one or two semesters, running from September to January and February to June, designed for highly recommended younger scholars, as well as for senior scholars with established reputations. Candidates who have not both finished their dissertations and had at least a year of full-time teaching experience are not eligible. Fellows are expected to live in Princeton, in order to take an active part in the intellectual interchange with other members of the Seminar. Funds are limited, and candidates are, therefore, urged to apply to other grant-giving institutions as well as the Center.

Inquiries and requests for Fellowship Application Forms should be addressed to the Secretary, Davis Center for Historical Studies, I29 Dickinson Hall, Princeton University, Princeton, New Jersey, 08540, U.S.A. The deadline for Fellowship applications and for letters of recommendation for the academic year $1975-76$ is December I, r974. Scholars who are not applicants for Fellowships but would like to visit Princeton to offer a paper to the Seminar are asked to write to the Director.

\author{
PRINCETON UNIVERSITY, Department of History \\ I29 Dickinson Hall \\ PRINCETON, New Jersey o8540
}


Vincent AURIOL

\section{JOURNAL DU SEPTENNAT}

version intégrale

\section{Parution du tome 2 : 1948}

édition établie et annotée par Jean-Pierre AZÉMA

$15,5 \times 24,776$ pages, h.-t., relie $-78 \mathrm{~F}$

\section{tome I : 1947}

édition établie et annotée

par Plerre NORA

$15,5 \times 24,948$ pages,

5 h.-t., relie - 89 F

\section{tome 7 : 1953-1954}

édition établie et annotée par Jacques OZOUF

$15,5 \times 24,912$ pages,

1 h.-t., relie - 83 F

\section{Dávid EASTON}

\section{ANALYSE}

DU SYSTÈME POLITIQUE

traduction de Plerre ROCHERON

Un livre à la fois célèbre et mal connu. Peu d'ouvrages de science politique ont suscité depuis une dizaine d'années une réflexion et des débats comparables. La présente traduction, augmentée d'une postface inédite de l'auteur, permettra au public françals d'accéder directement à une pensée dont la simplicité apparente n'exclut ni l'abstraction ni la complexité.

Collection " Analyse politique \#, 15,5 $\times 24,500$ pages - $95 \mathrm{~F}$ 


\title{
ANNALES DE BRETAGNE ET DES PAYS DE L'OUEST
}

\author{
(Anjou, Maine, Touraine)
}

Revue trimestrielle d'histoire publiée par les Universités d'Angers, Brest, Le Mans, Rennes II et Tours, avec le concours du C.N.R.S.

\section{4 - No 1}

L. FOUCHER, Sur l'image du mois de Mars.

M. GALIOU, Notes de céramologie armoricaine.

F. MORVANNOU, Guénolé et Guénaël.

$\mathrm{X}$. MARTIN, Pouvoir royal et finances urbaines au temps des guerres de Religion : deux épisodes de l'histoire angevine.

G. MILIN, Modèles idéologiques et modèles culturels dans l'auvre narrative de Noël du Fail.

J.-J. HURT, La politique du parlement de Bretagne (I66r-1675).

J. BRENGUES, La franc-maçonnerie dinannaise de 1760 à 1789 .

F. LEBRUN, Angers sous l'Ancien Régime : introduction à l'histoire démographique de la population.

H. CORBES, En parcourant les mémoires d'un administrateur de la marine au siècle dernier.

J.-R. CHOTARD, Évolution des mentalités dans l'ouest de l'arrondissement de SavenaySaint-Nazaire, de la Restauration à $1920, d^{\prime}$ après les procès-verbaux de visites pastorales.

Abonnement annuel : France, 5o F - Etranger, 6o F. Le numéro : I6 F.

Le montant des abonnements sera versé au compte suivant : Annales de Bretagne et des Pays de l'Ouest, C.C.P. Rennes 426-37 S, Université de Haute-Bretagne (Rennes II), avenue Gaston-Berger, 3500o Rennes.

\section{Annales du Midi}

Revue de la France Méridionale

UNIVERSITES DE BORDEAUX - TOULOUSE - MONTPELLIER

AIX-MARSEILLE - NICE - PAU - LIMOGES

Édouard Privat, éditeur - I4, rue des Arts, I4, Toulouse oI

TOME LXXXVI- 1974

Fascicule $I$ (janvier-mars) :

Gisèle BOURGEOIS, Formation et structure d'un village médiéval en Rouergue : SaintJean-d'Alcas.

Henri MICHEL, Urbanisme et société à Montpellier sous l'Ancien Régime. Un exemple : le sixain Saint-Firmin.

Michel DERLANGE, En Provence au XviII ${ }^{\mathbf{e}}$ siècle : la représentation des habitants aux conseils généraux des communautés.

Jean SAGNES, Un journal socialiste de l'Hérault : L'Étincelle (r898-1904).

Fascicule 2 (avril-juin) :

Jean-Baptiste ROBERT, Droits des seigneurs et frontière politique : La Turbie, Roquebrune et Menton du $\mathrm{XII}^{\mathbf{e}}$ au $\mathrm{XV}^{\mathbf{e}}$ siècle.

Béatrice LEROY, Une bastide frontière navarraise du xrve siècle : Echarri-Aranaz.

Claude C. STURGILL, Une source de l'histoire sociale urbaine : le rôle des miliciens de Bordeaux de I 722 .

Roger CORNU, Les portefaix et la transformation du port de Marseille.

$$
\begin{gathered}
\text { Abonnements : France : } 40 \mathrm{~F} \longrightarrow \text { Etranger : } 50 \mathrm{~F} \\
\text { Prix du numéro : I2 F }
\end{gathered}
$$




\section{ÉCONOMIE INTERNATIONALE}

\section{Tome I. Economie financière}

\section{Pascal SALIN}

Une méthode d'analyse qui permet d'aborder, et de comprendre pour tenter de les résoudre, tous les problèmes de l'économie monétaire internationale.

Collection U, $17 \times 23,288$ pages $-38 \mathrm{~F}$

\section{EUROPE :}

\section{VERS UNE POLITIQUE CONJONCTURELLE COMMUNE}

\section{Paule ARNAUD-AMELLER}

II seralt nécessaire et urgent de pratiquer une politique d'expansion commune qui solt mise en ceuvre par des moyens appropriés. La première démarche pourrait emprunter la vole d'une politique budgétaire commune.

"Travaux et Recherches de Sciences Economiques "1, 15,5! $\times 24,126$ pages - $38 \mathrm{~F}$

\section{armand colin}




\section{THE \\ ECONOMIC HISTORY \\ REVIEW}

Second Series, Volume XXVII, No. 2, May 1974

\section{CONTENTS}

\section{ARTICLES}

CHRISTOPHER CLAY

The Price of Freehold Land in the Later Seventeenth and Eighteenth Centuries

CHARLES K. HYDE

Technological Change in the British Wrought Iron Industry, I750-1815:

$A$ Reinterpretation

PETER TEMIN

The Anglo-American Business Cycle, $1820-60$

PAUL L. ROBERTSON

Technical Education in the British Shipbuilding and Marine Engineering

Industries, I863-I9I4

PETER FEARON

The British Airframe Industry and the State, I9I8-35

LESLIE HANNAH

Managerial Innovation and the Rise of the Large-Scale Company

in Interwar Britain

COMMENT

DEREK H. ALDCROFT

McCloskey on Victorian Growth: A Comment

DONALD N. MCCLOSKEY

Victorian Growth: $A$ Rejoinder

N. B. HARTE and D. J. TIERNEY

List of Publications on the Economic and Social History of Great Britain and Ireland published in 1972

\section{REVIEWS}

Published for The Economic History Society by

The Broadwater Press Ltd, Welwyn Garden City, Hertfordshire, England 


\title{
ÉCOLE PRATIQUE DES HAUTES ÉTUDES - VIe SECTION SCIENCES ECONOMIQUES ET SOCIALES \\ Centre de Recherches Historiques
}

\author{
A L A I N C R O IX \\ NANTES \\ ET LE PAYS NANTAIS \\ étude démographique
}

Cette étude régionale, une des premières du genre sur le Xvi siècle, utilise largement l'inestimable trésor des registres paroissiaux bretons. Complétés par le dépouillement d'archives très diverses, ils permettent de faire revivre de multiples aspects de la manière de vivre de nos ancêtres : amour, nourriture, maladie, mort, religion, ces mots-clés de la vie quotidienne de l'Ancien Régime, sont abordés sous des angles suffisamment variés pour que les distances entre groupes sociaux, entre ruraux et citadins en ressortent nettement.

1974, 357 pages, 6o $\mathrm{F}$ (Démographie et sociétés, I5).

\section{S E V P E N}

I3, RUE DU FOUR, 75006 PARIS 


\section{REVUE TIERS-MONDE}

Tome XV, n० 57

Janvier-Mars 1974

POUVOIR, MYTHES ET IDÉOLOGIES

sous la direction du Professeur Guy CAIRE

\section{PERTINENCE DE L'IDÉologie}

Guy CAIRE, Idéologies du développement et développement de l'idéologie.

Georges LABICA, Pour une approche critique du concept d'idéologie.

Jacques AUSTRUY, La transformation du pouvoir dans le début du développement économique. Celso FURTADO, Le mythe du développement et le futur du Tiers-Monde.

Guy de LACHARRIËRE, Aspects mythiques des doctrines relatives au commerce international. Jean MOULY, L'emploi dans la pensée relative au développement économique.

Odette GUITARD, Organisation politique du Tiers-Monde de Bandoung à Santiago.

Gérard DESTANNE de BERNIS, Le sous-développement, analyses ou représentations.

\section{LES IDÉOLOGIES MOBILISATRICES}

Yves BENOT, Idéologies, nation et structures sociales en Afrique noire.

Tomas Amadeo VASCONI et Marco Aurelio GARCIA de ALMEIDA, Le développement des idéologies dominantes en Amérique latine.

Jan DELEYNE, Idéologie et développement en Chine Populaire.

Maria Isaura PEREIRA de QUEIROZ, Mythes des paysans brésiliens.

Alain TOURAINE, Mouvements sociaux et idéologies dans les sociétés dépendantes.

Christian PALLOIX, Impérialisme et mode d'accumulation international du capital. Essai d'une approche du néo-impérialisme.

Yves LAULAN, Les nouveaux mythes: pollution et environnement.

Direction-Rédaction : Institut d'Étude du Développement Économique et Social, 58, boulevard Arago - 75013 PARIS - Tél. : 331-28-01

Abonnements et vente: Presses Universitaires de France, 12, rue Jean-de-Beauvais 75005 PARIS - Tél. : 033-48-03 - C.C.P. : PARIS n 1302-69

France : F. 76,00

Étranger : F. 85,00 


\section{ÉCOLE PRATIQUE DES HAUTES ÉTUDES VIe SECTION - SCIENCES ÉCONOMIQUES ET SOCIALES}

Contributions du Centre d'Études Arctiques Io.

RÉGIS BOYER

\section{LE LIVRE \\ DE LA COLONISATION DE L'ISLANDE \\ (LANDNAMABÓK)}

Introduction, traduction, notes et commentaire de Régis Boyer.

Le Livre de la colonisation de l'Islande, œuvre originale et unique en son genre, datant de la fin du XIII ${ }^{\mathbf{e}}$ siècle, est proposé ici pour la première fois en traduction française, allégée des généalogies fastidieuses et selon une méthode de présentation qui permette d'en confronter les différentes versions. La traduction est accompagnée de notes explicatives, précédée d'une introduction historique et critique, suivie d'un essai d'étude de la civilisation nordique ancienne.

1973, I69 pages, $45 \mathrm{~F}$.

\section{MOUTON}

Diffusion pour la France:

LIBRAIRIE DE LA NOUVELLE FACULTÉ

30, RUE DES SAINTS-PÈRES, 75007 PARIS 


\section{ACTA POLONIAE HISTORICA}

Benedykt ZIENTARA

Jakub SAWICKI

Michał TYMOWSKI

Krzysztof GAWLIKOWSKI

Zbigniew LANDAL

Marian BISKUP Jerzy MATERNICKI

Czeslaw MADAJCZYK

Władyslaw CZAPLIŃSKI et Wojcieciı WRZESIŃSKI

Tadeusz GRUDZIŃSKI Stanisław RUSSOCKI Jerzy MICHALSKI

Ludwik HASS

Jerzy W. BOREJSZA Józef CHLEBOWCZYK

Wiesław ŚLADKOWSKI

Leon GROSFELD

Aleksander GIEYSZTOR

\section{VOLUME XXIX}

\section{Études}

Foreigners in Poland in 10th-15th Centuries : Their Role in the Opinion of Polish Medieval Community.

Ein unbekanntes Konkordat ? (Ein Beitrag zur Geschichte der Beziehungen zwischen Staat und Kirche in Polen zur Zeit der Reformation). La ville et la campagne au Soudan Occidental du $\mathrm{xrv}^{\mathbf{e}}$ au $\mathrm{xvI}^{\mathbf{\theta}}$ siècle (problème des rapports économiques).

The Chinese War-lord System of the 1920s. Its Origin and Transformations.

Nouvelles méthodes

Comparative Research on the long-range economic Growth of Poland (a Proposal concerning the selection of States for Comparison).

\section{Matériaux}

En marge des grands anniversaires : Copernic 1473-1973

(Commission d'Education Nationale 1773-1973).

Copernicus' Verhältnis zum Deutschen Ritterorden.

L'enseignement de l'histoire en Pologne au xviII' siècle.

Recherche historique : Courants et structures

Les vingt ans de l'Institut d'Histoire de l'Académie Polonaise des Sciences (bilan et perspectives).

Discussion

The Polish Western Frontier.

Comptes rendus

Notes

Vie scientifique

Controverses autour de la "Drôle de Guerre "(Tadeusz JFDRUSZCZAK) Rencontre internationale d'historiens de la Seconde Guerre Mondiale (Stanislawa LEWANDOWSKA).

\section{VOLUME $\mathrm{XXX}$}

Etudes

The beginnings of feudal division in Poland.

Les assemblées pré-parlementaires en Europe Centrale.

L'opposition capitale-province et ville-campagne dans la mentalité des Polonais de la seconde moitié du xyırre siècle.

The Socio-Professional Composition of Hungarian Freemasonry (18681920).

Essais

Portrait du révolutionnaire polonais.

Die Madjarisierungs- und die Germanisierungspolitik im 18.-19. Jahrhundert und um die Jahrhundertwende. Versuch einer Konfrontation. Matériaux

La gauche française et la question de l'indépendance de la Pologne au cours de la Première Guerre Mondiale.

La Pologne face au problème de l'évacuation de la Rhénanie (1926-1929). Recherche historique : Courants et structures

L'Histoire à l'Université de Varsovie.

Comptes rendus

Notes

Vie scientifique

In Memoriam : Stanislaw Herbst (Wladyslaw TOMKIEW ICZ). Chronique.

\section{INSTYTUT HISTORII PAN}

Warszawa, Rynek St. Miasta 29/31

Pour commande à l'étranger s'adresser à la Centrale du Commerce Extérieur ARS POLONA

Warszawa, Krakowskie Przedmiescie 7 


\author{
ÉCOLE PRATIQUE DES HAUTES ÉTUDES \\ VIe SECTION - SCIENCES ÉCONOMIQUES ET SOCIALES \\ Société, mouvements sociaux et idéologies \\ Études, I5
}

RENÉ D UCHAC

\title{
LA SOCIOLOGIE DES MIGRATIONS AUX ÉTATS-UNIS
}

Une synthèse, originale en France, du développement de la sociologie urbaine américaine, dans sa relation aux problèmes des relations interethniques et interraciales. L'étude des faits migratoires, entreprise à tous les niveaux - du mathématique au politique - sert d'axe directeur à l'analyse d'un fait majeur du changement social aux États-Unis : la construction d'une société fondée sur la reconnaissance du pluralisme culturel.

I974, 656 pages, $78 \mathrm{~F}$.

\section{MOUTON}

Diffusion en France:

LIBRAIRIE DE LA NOUVELLE FACULTÉ

30, RUE DES SAINTS-PÈRES, 75007 PARIS 


\section{L'homme et la société}

Revue internationale

de recherches et de synthèses sociologiques

\section{EDITIONS ANTHROPOS}

Direction-Rédaction-Administration-Abonnements

12, avenue du Maine, Paris 15, Tél. 548-42-58 - 222-76-82

Numéro double

Juillet-Août-Septembre 1973

Nos 29-30 Octobre-Novembre-Décembre 1973

\section{ANALYSE INSTITUTIONNELLE GÉNÉRALISÉE}

Jean-Marc SALMON : Le désir du 22 mars.

René LOURAU: Analyse institutionnelle et question politique.

Rémi HESS: Le maoisme, l'analyse et les analyseurs.

Cynthia HAFT : L'institution totalitaire - le moral et la morale dans les camps de concentration nazis.

Michel BERNARD : Les conditions du groupe d'action.

Pierre EVRARD : Les mains pures.

Yves STOURDZE : Espace, circulation, pouvoir.

Antoine SAVOYE: Pour une analyse institutionnelle du parti.

\section{LE CORPS}

Georges LAPASSADE: Le mouvement du potentiel humain. Max PAGES : La libération du corps.

Jean-Marie BROHM : Vers l'analyse institutionnelle du sport de compétition.

\section{LA SOCIANALYSE}

Patrice VILLE : L'analysme.

René LOURAU: Monographie d'une intervention socianalytique - déplacements à Louvain.

Laurence GAVARINI et Antoine SAVOYE : La socianalyse en question une intervention socianalytique à l'Université Libre de Bruxelles.

Rémi HESS : L'institution de l'inadaptation - une intervention socianalytique dans une école d'éducateurs spécialisés.

Georges LAPASSADE : La rencontre institutionnelle.

Jean-Marie VINCENT : Hommage à Serge MALLET.

\section{NOTES CRITIQUES}

Alain GUILLERM : Le retour de " Socialisme ou Barbarie ". Jacques GUIGOU : Socianalyse de l'éducation permanente des socialistes. J. F. de RAYMOND : Le théâtre de la spontanéité (Moreno). 


\section{ÉCOLE PRATIQUE DES HAUTES ÉTUDES \\ VI. SECTION - SCIENCES ÉCONOMIQUES ET SOCIALES \\ Centre de Recherches Historiques}

M A R C E L L O C A R M A G A N I

\section{LES MÉCANISMES \\ DE LA VIE ÉCONOMIQUE \\ DANS UNE SOCIÉTÉ COLONIALE : \\ LE CHILI, I680-I830}

L'étude des mécanismes de la vie économique du Chili offre à l'auteur l'occasion de poser quelques problèmes fondamentaux de la croissance économique des colonies espagnoles en Amérique. A travers l'analyse des mécanismes qui règlent le commerce extérieur, les échanges internes et la production agricole et minière, il montre que dès le Xvin ${ }^{e}$ siècle la formation d'un marché "national " permet aux puissances européennes de contrôler une économie en constant déséquilibre, processus qui favorisera l'apparition d'un véritable sous-développement.

1973, 393 pages, 98 F (Monnaie - Prix - Conjoncture, Ir).

\section{SEVPEN}

I3, RUE DU FOUR, 75006 PARIS 


\title{
RAISON PRÉSENTE
}

REVUE TRIMESTRIELLE DE CRITIQUE ET DE SYNTHESE ( $7^{\mathrm{e}}$ année)

\author{
Directeur : Victor LEDUC
}

SOMMAIRE DU No 30 - AVRIL, MAI, JUIN 1974

\section{Éditorial}

Victor Leduc, La vérité en exil.

\section{Études}

Jean Piaget, Histoire et développement de la causalité.

Maurice Caveing, L'automate et la pensée.

Denise Dumont-Dressy, Les Protestants et l'acuménisme.

Gabriel GoHau, École et société.

Charles MAIGNIAL, Histoire, image et vérité à propos de "Français si vous saviez ".

Michael Spencer, Arthur Rimbaud ou le Monde renversé.

\section{Trimestrielles}

Michel Rouze, Autour de la Science.

Maurice Audebert, Théâtre.

Jeanne Brunschwig, Carmel Samut, Livres.

\section{Éditions Rationalistes}

I6, rue de l'École Polytechnique - 75005 PARIS

C.C.P. PARIS I8.378.84 Tél. : 633.03 .50

Abonnement annuel (4 numéros); France $38 \mathrm{~F}$ - Étranger $42 \mathrm{~F}$

Le numéro $11 \mathrm{~F}$ 


\section{Ideologie}

\section{JOSÉ MARTÍ}

Volumi speciali di Ideologie, con illustrazioni a colori, interamente dedicati all'Apostolo cubano.

Volume I: Scritti di José Marti (pagine 376), preceduti da alcune pagine di Fidel Castro e da una Introduzione di Cintio Vitier :

CUBA : Il carcere politico a Cuba ; Con tutti e per il bene di tutti; Difesa di Cuba ; Principi del Partito Rivoluzionario Cubano ; Le nostre idee ; Il rimedio annessionista ; La mia razza; La crisi e il Partito Rivoluzionario Cubano; Il terzo anno del Partito Rivoluzionario Cubano ; Il ro aprile ; Il Generale Gómez ; Manifesto di Montecristi ; Lettera a Manuel A. Mercado.

LA NOSTRA AMERICA : Madre America; La nostra America; Discorso su Bolívar ; Il Congresso Internazionale di Washington, I, II ; La Conferenza Monetaria delle Repubbliche d'America; L'uomo antico d'America e le sue arti primitive; Autori americani aborigeni; Le rovine indie; Lettera a Federico Henríquez y Carvajal.

STATI LNITI : Emerson; Il poeta Walt Whitman; Un dramma terribile; L'eccidio degli italiani; La verità sugli Stati Uniti.

Scelta dai DIARI, dalle LETTERE e dalle POESIE.

Volume II : Scritti su José Martí (pagine 344).

A) L'OPERA POLITICA E SOCIALE : Julio Antonio Mella, Commentando $i$ pensieri di José Martí (I926), Emilio Roig de Leuchsenring, Martí antimperialista (1953) ; José Antonio Portuondo, Introduzione allo studio delle idee sociali di Martí (I942) ; Julio Le Riverend, Martí, etica e azione rivoluzionaria (I969) ; Roberto Fernández Retamar, Note su Marti, Levin e la rivoluzione anticoloniale (1970).

B) L'OPERA LETTERARIA : Federico de Onís, José Marti, valutazione (I952) ; Rubén Dario, José Martí (I895); Miguel de Unamuno, Sullo stile di Marti (I9I9); Gabriela Mistral, La lingua di Marti (1934); Juan Ramón Jiménez, José Martí (1938) ; Manuel Pedro González, Marti, creatore della grande prosa modernista (1953) ; Juan Marinello : Marti : poesia (rg68).

C) L’UOMO : Ezequiel Martínez Estrada, Il diario di campagna di Martí come documento caratterologico (I963); Gonzalo de Quesada y Miranda, Tavola cronologica della vita di José Martí (I960).

D) Bibliografia martiana, a cura di Cintio Vitier.

NOTA : Il Volume I viene inviato agli abbonati per il 197 I quale fascicolo $18^{\circ}-19^{\circ}-$ $20^{\circ}$ della rivista, a conclusione dell'annata 197 I e come omaggio della prima metà dell'annata 1972 ; il Volume II, quale fascicolo $2 I^{\circ}-22^{\circ}$, viene inviato, insieme al I, agli abbonati per il $197^{2}$, concludendo formalmente tale annata arretrata.

Non si accettano più abbonamenti. - Sono disponibili i volumi arretrati.

Via Girolamo Segato 3I, ool47 ROMA.

C.c.p. I / I 229 intestato a Ideologie;

c.c. ${ }_{5} 67$, Banca Nazionale del Lavoro, Ag. 19, ROMA. 


\section{DIOGÈ NE}

REVUE INTERNATIONALE DES SCIENCES HUMAINES

PUBLIÉE AVEC L'AIDE DE L'UNESCO

Rédacteur en chef : Roger CAILLOIS - Secrétaive de rédaction : Jean d'ORMESSON

$$
\text { No 86. - AvrIL-JuIN I974 }
$$

Jacques BERQUL

Takeshi LMEHARA

Jacques BINET

Olivier REBOUL

Alexandre MikhaïLov

Jacob Brovowski

Mauricio OBREgON

Stan J. SCOTT
L'algébrique et le vécu

La généalogie des esprits vengeurs

Drogue et mystique : le bwiti des Fangs

Slogans et enseignement

Rhétorique allemande du xviı ${ }^{e}$ siècle

\section{CHRONIQUES}

L'expérience créatrice

Des Argonautes aux astronautes

La mythologie romantique du langage

Rédaction et Administration (Nouvelle adresse) :

U.N.E.S.C.O., I, rue Miollis, 750 I 5 Paris. Tél. 566-57-57

Revue trimestrielle paraissant en quatre langues: anglais, arabe, espagnol et français

L'édition française est publiée par la Librairie Gallimard,

5 , rue Sebastien-Bottin, 75007 Paris

Les abonnements sont souscrits auprès de cette maison (C.C.P. 169-33 Paris)

Prix de vente au numéro: $12 \mathrm{~F}$

Tarifs d'abonnement: France et Communauté, $36 \mathrm{~F}$; Étranger, $41 \mathrm{~F}$

\section{REVUE ÉCONOMIQUE}

Publication bimestrielle

avec le concours de la VI' Section de l'École Pratique des Hautes Études et du Centre National de la Recherche Scientifique

Jacques LECAILLON, La hiérarchie des salaires. Réflexions sur l'expérience française.

Alain d'IRIBARNE, L'action du progrès technique sur l'évolution des qualifications.

Gérard LAFAY, Spécialisation internationale et croissance nationale : une approche par la théorie des créneaux.

Daniel BREFORT, Le comportement d'investissement des entreprises: quelques nouveaux résultats.

Dominique VALLET, Présentation résumée du modèle CANDIDE.

Rémy PRUD'HOMme et Philippe ROCHEFORT, Pollution et dépollution optimales : le cas des automobiles dans une grande agglomération.

Roger GUESNERIE, Environnement et redistribution : une note.

Christian PETIT, Un test économétrique de l'analyse keynésienne de la demande d'encaisses.

Abonnements 1974 : France $100 \mathrm{~F}$. - Étranger : $120 \mathrm{~F}$. Le numéro $18 \mathrm{~F}$.

Librairie A. COLIN, 103, boulevard Saint-Michel, 75240 Paris Cedex 5 - C.C.P. Paris 21335-25 
COLLOQUES INTERNATIONAUX

$\mathrm{DU}$

CENTRE NATIONAL DE LA RECHERCHE SCIENTIFIQUE

No 549

\section{LA CROISSANCE \\ DE LA GRANDE FIRME MULTINATIONALE}

Les textes, en français et en anglais, abordent les principaux problènes soulevés par la croissance de la grande entreprise multinationale. Trois aspects sont mis en évidence : les causes et le repérage de cette croissance, ses modalités, ses conséquences.

Les communications vont de l'analyse théorique à la présentation de situations concrètes. Cependant l'accent a été mis sur les difficultés que soulève la connaissance des sociétés multinationales et de leurs effets sur l'économie.

\section{Ouvrage i6 $\times 25$, de 636 pages, broche \\ CODE (à rappeler) : 730084}

PRIX FF : 90,30

\section{ÉDITIONS DE}

\section{CENTRE NATIONAL DE LA RECHERCHE SCIENTIFIQUE}

I5, quai Anatole-France, 75700 Paris

Tél. $555 \cdot 26 \cdot 7^{\circ}$

C.C.P. PARIS g06I-II 


\section{TERZO MONDO}

rivista trimestrale di studi, ricerche e documentazione

sui paesi afro-asiatici e latino-americanj

diretta da Umberto MeLotti

anno VII (1974)

n. 23 (speciale)

\section{LA LEZIONE DEL CILE}

scritti e interventi di André Gunder Frank, Fernando H. Cardoso, Eriberto Torres Rivas, Antonio Carlo, Giampiero Cotti Cometti, Vittorio Dini, Elias Condal, Tullo Vigevani e Umberto Melotti.

Questo numero : L. I.200 (U.S.\$2) - Abbonamento I974 : L. 4.400 (U.S.\$8)

Collezione completa dal n. I (Ig68) al n. 22 (I973) : L. I5.000 (U.S.\$ 25).

\section{QUADERNI DI TERZO MONDO}

Da quest'anno a "Terzo Mondo "s'affianca una nuova collana di quaderni monografici. Sono già apparsi :

"Marx e il Terzo Mondo "

"Marx e il Mondo Antico "

di Umberto Melotti

di Leone Iraci

L. $\mathrm{I} .800$ (U.S.\$3)

"Lévi-Strauss e il Terzo Mondo "

di Petro Scarduelli

L. I.800 (U.S.\$3)

"La Tanzania verso il socialismo "

di G. P. Cotti Cometti

L. I.800 (U.S.\$3)

I quattro quaderni già pubblicati

Abbonamento ai prossimi quattro quaderni : L. 6.000 (U.S.\$ Io).

\section{CORSO DI SOCIOLOGIA}

\section{a dispense}

Finalmente a dispense il corso di formazione in sociologia organizzato dall'Istituto di Studi e Indagini Sociologiche e dal Centro Studi Terzo Mondo. La proposta di una sociologia critica ed impegnata nei testi di Umberto Melotti, Giuliano della Pergola, Giovanni Bianchi, Gino Del Grosso, Eugenio Turri, Alberto Merler, Tito Perlini, Adele Faccio e altri qualificati studiosi.

Abbonamento con diritto a tutti gli arretrati : L. I2.000 (U.S.\$20).

Ogni versamento relativo a "Terzo Mondo ", ai quaderni e al corso di sociologia va effettuato sul conto corrente postale n. 3/56III intestato a "lerzo Mondo ", Via G. B. Morgagni 39 - 20129 Milano. A tutti gli abbonati delle riviste che publicano il presente annuncio si concede lo sconto speciale del $20 \%$ sulle quote indicate. 


\title{
COMITÉ INTERNATIONAL DES SCIENCES HISTORIQUES \\ 270, Boulevard Raspail - 750I4 PARIS - Tél. : 033-26-29
}

\section{CONGRÈS INTERNATIONAL DES SCIENCES HISTORIQUES A SAN FRANCISCO}

\begin{abstract}
Répondant à l'invitalion qui Jui en avait élé faite par l'American Historical Association, l'Assemblée générale du 15 aont 1970 a décide que le X IV e Congrès inlernational des sciences hisloriques se tiendrait en 1975 aux ftats-Unis. Dès 1971 , dans sa réunion de Dublin, le Bureau du C.I.S.H. s'est préoceupe de définir les principes généraux et d'clablir le plan d'ensemble du Congrès, lesquels ont été soumis à l'Assemblée génćrale des $21-22$ juillet 1972 et adoptés par elle. L'American Historical Association avait précisé cntre-temps que le Congrès aurait lieu à San Franciseo du dimanche 22 au dimanche 29 aont 1975 inchus.

On trouvera ci-dessous l'essenticl des dispositions qui ont été retenues par le Conité organisateur du Congrès, lequel est composé du Bureau du C.I.S.H. et du Bureau de l'American Hislorical Association, et le programme d'ensemble du Congrès hui-mêne dont la struciure définitive a été arrêtée par lé Comité organisateur en avril 1973
\end{abstract}

\section{DISPOSITIONS GERERALES}

1.0 XIV: Congrès intermational des sciences historiques qui se tiendra à San francisco du 22 au 29 aont 1975 comportera quatre sections qui seront consacrees respectivenent : $a$ ) aux grands thèmes d'ćtude ; b) aux problemes de méthodologic ; $c$ ) à l’histoire par périodes chronologiciues ; $d$ ) aux travaux des Organismes internationaux aftiliés et des Commissions qui seront intégrés dans le programme du Congrès.

Les six rapports de synthèse qui seront présentés daus les deux premières sections seront confiés, pour leur élaboration, à la respousabilité d'un Comité national ou d'un Organisne internationa] affilié. Ene journée entière sera consacrée à leur discussion, mais ils seront seuls à faire l'objet d'un débat entre experts au cours de la matinée, la discussion étant ouverte à tous les congressistes dans l'après-midi.

Dans la troisième section, deux et éventuellement trois questions seront traités à l'intérieur de chacune des quatre grandes périodes; pour chacune des questions retenues, un certain nombre de rapports seront présentés à titre individuel mais sous le couvert d'un Comité national. La discussion de ces rapports aura lieu seulement l'après-midi, de façon à laisser aux congressistes la possibilité d'assister le matin aux séances de discussion avec experts des deux premières sections.

Les Organismes internationaux affiliés et les Commissions dont les travaux ont ćlé intégrés dans le Congrès pour en constituer la quatrième section auront toute latitude pour assurer à leur gré la préparation de leur rapport à la discussion duquel sera consacrce une journce entière.

Les rapports, dont la longueur respective sera précisće ci-dessous, devront parvenir à l'Antcrican llistorical Associalion (400 A Street, S.E., Washington D C 20003) pour le ter octobre 1974 au plus tard.

Les rapports seront difusés avant le Congrès par Ies soins de l'American Ilistorical Association qui ies enverra en nombre, sous forme de phofocopies aux Comités nationaux, lescuels en assureront la réparlition dans leur pays respectifs.

Le choix des rapporteurs est laissé aux Comités nationaux et aux Organisnes internationaux affilics engagés dans la préparation du Congrès. Ils devront en informer au plus tól Je Secrétaire général du C.I.S.H. auquel ils devront également adresser leurs propositions pour la désignation des experts et des présidents el vice-présidents de séances; ces propositions auront à être faites en 1974 et pour le 30 juin at plus tard.

Ie droit d’inseription sera en principe de 25 dollars mais pourra faire l'objet de taux diflérenciés suivant les cas cule le Comité organisateur du Congrès se réserve de déterminer.

PROGRA M M E

\section{Première Section}

GRANDS THİMES D'ÉTLDE

1. Science historique el sociéti (L.R.S.S.).

2 . Les Droits de l'homme (France)

3. l.es Révolutions (U.S.A.).

4. Les Minorités (Canada).

5. I.es Migrations (Commission intern. pour l'élude des mouvements sociaux et des stractures sociales).

6. Traditions et innovations en Asie et en Afrique (Japon). 


\section{Deuxième Section}

Problèmes de méthodologie

1. Evénement, évolution et structure dans I'I Iistoire (Allemagre D.L.R.).

2. Les problèmes des "jugements de valeur "dans les sciences historiques (Prus-Bas).

3. L'historiographie comme science historique (Italie).

4. L'historien à la recherche de sa documentation (Pologne)

5. Méthodes de l'étude de l'homme dans son environnement (Suède).

6. Publication de documents et diffusion des sources (Allemagne R.F.A.).

Troisieme Section

ITSTOIRE PAR péRIODES CHRONOLOGIQTES

\section{ANTIQUITE :}

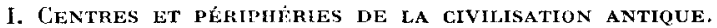

1. Die Beziehungen der griechisch-römischen Antike zum mittleren und ostlichen Sưasien (araf)ischer, iraniseher und indischer Raum), als Beitrag zum Universal-Geschiehte (Allemagne R.F.A.)

2. Les peuples balkaniques anciens (Thraces, Illyriens, etc.), leur rôle et importance dans l'évolulion historique du monde antique (Bulgarie).

3. Centres et périphéries de la civilisation antique (Pologne).

4. Greece and the Orient in historical times (Chypre).

5. Les provinces et les "limes " (IIongrie).

6. The case of China, Korea and Japan (Corée du Sud).

Il. 'TYPEs DE SOCIÉtés DANS L'ANTIQUité.

1. Les types de communaulés dans le monde antique (U.R.S.S.).

2. Aspectos económicos y demográficos en la colonización fenicia (tispagne).

3. La crise de la Polis athénienne au Ive siècle (Tchécoslonaquie).

4. Urbanisation als Phänomen der Antike (Allemagne R.F.A.).

5. Le role des "Kouchans" dans la civilisation mondiale (L.R.S.S.).

6. The urban revolution in a socio-political perspective (I .S.A.).

\section{MOYEN AGE :}

I. LES SOCIÉTÉS NOMADES.

1. Populations nomades dans l'espace euro-asiatique el leur rôle dans la formation des ćlats médiévaus (Foumanie).

2. Nomadic society (settled) agricultural society in the history of Asia (Japon).

3. Nain characters of leudalism of the nonades (Mongolie)

4. Der mongolische Nomadismus in einer seeshaften Gesellschaft : die goldene Horde (R.F.A.).

5. Orient et Occident dans l'histoire médiévale du Sud-Est européen (Yougoslavie).

6. Le nomadisme en Amérique jusqu'au début du xvir siècle (Mexique).

II. HENCONTrES DE CIVILISATIONS EN EUROPE VERS 1300.

1. Structures sociales el politiques de l'Europe du xirie siècle (Pologne).

2. liencontres entre peuples slaves et peuples d'Orient vers 1300 (T'chécoslovaquie)

3. Les universités du bassin méditerranéen du milieu du xrIte à la fin du xive siecle (Suint-Siège)

4. Ėl mundo mediterraneo en torno al año 1300 (Espagne, Grèce, Italie).

5. Les Juifs et le monde méditerranéen vers 1300 (Israél)

6. Cites et langages en Europe centro-orientale (Hongrie).

7. " Expetling" the Muslims fron Spain : the demographic changes ( $U$.S.A.).

\section{HISTOIPE MODIENE :}

I. Nations ET ÉTATS ( $\mathrm{XVI}^{\mathrm{e}} \mathrm{- \lambda VII}{ }^{\mathrm{e}}$ siècle).

1. Ia formation d'Fiats centralisés en Europe à la fin du Moyen Age (L.R.S.S.).

2. Les compétences de l'Etat y compris la liscalité publique (France).

3. Religion and society in Reformation Europe (Grande-Bretagne)

4. La politique ot tomane dovant la Réforne (Turquie).

5. National stability and hereditary transmission of political and economic power (U.S.A.).

6. L'expansion ottomane et l'essor des Etats centralisés en Europe de l'Est (Hongrie).

7. Land. Staat und Reich im politischen Bewusstsein deutseher Landstände im 18. Jhrdt (K.F.A.).

II. Aspects Économiques des sociétes EN Díveroppenent industriel (Xvite-Xixe siècle).

1. Optimisme et pessimisme dans l'interprétation des effets sociaux de la révolution industriclle en GrandeBretagne (lin xvir et première moitié du xix ${ }^{e}$ siècle) (Norvège).

2. Employment and unemployment in the 19th century (Danemark)

3. Le processus de la modernisation pendant les $x$ vile-xixe siècles dans les sociétés de l'lírope de l'Est (Roumanie).

4. Fconomic development, imperialism and the taxpayer : a study of social choice in Britain and welfare in the colonies (U.S.A.).

5. Interuationaler Finanzimperialismus vor 1914 (R. $I^{*}, A$, )

6. Illuminismo e riforme net settecento italiano e spagnolo ; convergenze e divergenze (Italie).

7. I a première rivolu? ion industrielle el les problèmes de l'asynchronisne de l'évolution sociale (Polonıe). 
IISTOIRE CONTEMPOHAINE :

I. L'EuRope er les ETats-Unis d'Amérique.

1. Beziehungen zwischen den europäischen Fortschriltskräften und den C.S.A. im 19. Jahrhundert (Allemagne D.D.R.).

2. IAmérique russe el les relations russo-américaines au cours des Xvir ${ }^{\mathrm{e}}$ et $\mathrm{xix}^{*}$ siècles (Tchécoslovaquie).

3. L'instauration des rapports diplomatiques entre les États-Lnis de l'Amérique du Nord el les Ftats européens aux Xvir ${ }^{\mathrm{e}}$-XIX ${ }^{\mathrm{e}}$ siècles (Roumanie).

4. Der deutsche Frühkonstitutionalismus und das amerikanische Vorbild (K.F.A.).

5. Les L.S.A. et l'Lurope centrale entre les deux guerres mondiales (Pologne).

11. LE PHOBLEME DE LA DIMOGRATIE DANS IA STRICTLRE INTERNE DES PARTIS ET MOLVENENTS POLITIQUES AU Xxe SUT:CLE.

1. Le probleme du "Leadership o dans les partis politiques des démoeraties de l'Europe occidentale au $\mathrm{xx}^{\mathrm{e}}$ siècle (Suisse).

2. Socialist revolution or bourgeoise democratic reform in the labour movement in the desintegrating austro-hungarian monarchy (IIongrie).

3. Das Problem der Demolsatie in kleimbürgerlichen Verbänden und Bewegungen im ersten Drittel des 20. Jahrhunderts : Forschungstand und Forschungsperspektiven $($ R.I.A.).

4. The elite-mass nexus in African nationalist movements in the xxth century ( $L$.S.A.).

5. Principes démocratidues et principes socialistes dans les programmes des partis ouvriers (l'R.S.S.).

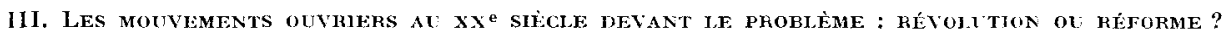

1. Révolution ou réforme dans les partis de la I)euxième Internalionale en Europe occidentale (Hongrie).

2. Die Arbeilerbewegung in Mitteleuropa vor der Frage : Reform oder Revolution 1914-1919? (IR.F.A.).

3. Revolution ou réforme en Europe centrale au $\mathrm{xx}^{\mathrm{e}}$ siecle (Pologne).

4. Class, mion and party in Western Europe (U.S.A.).

7. Der Lormierungsprozess der Arbeitcrklasse in der zweiten Hälfte des xix, urd zu Beginn des xx. Jahrhunderts $(D, D, R$.).

b. Les mouvenments ouvriers dans les Balkans devant le problème : révolution ou réforme (Yougoslavie).

IV. IDÉES E'T REALITF́:S POLITIQUES At' $\mathrm{xX}$ SIÈCLE.

1. The breakdown of the european security system 1980-1939 (Grande-Bretagne).

2. Les origines de la notion de coexistence (U.R..SS.).

3. Leadership and transition from the politics of revolulion to the politics of party: the example of ireland 1914-1939 (Irlande).

\section{Quatrième Section}

\section{ORganismes internationaty apfitiés ET Gommissmons}

1. Association internationale d'histoire du droit et des institutions :

La pénétration des droits étrangers dans les droits nationaux et locaux depuis le xvie siècle (EuropeAm(rique).

2. Commission internationale d'histoire des villes :

Morphologie urbaine et structures sociales dans le développement historique des villes.

3. Commission internationale d'histoire économique :

Production et commerce mondiaux de l'or, de l'argent ef du cuivre au début de l'époque moderne (1450-1750).

4. Fédération internationale des sociétés et instituts pour l'étude de la Renuissance : Humanisme, religions et utopies sociales à l'ćpoque de la Renaissance.

\section{$*^{*} *$ \\ NUTE SIR LA PUÉSENTATION DES RAPPORTS.}

Grants thèmes d'étude : 80 pages imprimées pour chacun des rapports de synthèse, à l'exception du rapport sur "les Migrations" qui en comportera 120.

problème de méthodologie: 32 pages imprimées par rapport.

Histoire par périodes chronologiques : 15 pages par rapport.

organismes internationaux affiliés et commissions :

Ilistoire du droit et des institutions $\ldots \ldots \ldots \ldots \ldots \ldots \ldots \ldots \ldots \ldots \ldots \ldots \ldots \ldots \ldots \ldots \ldots, 64$ pages

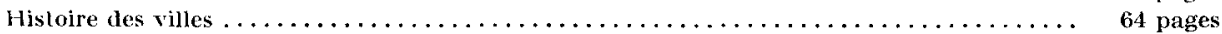

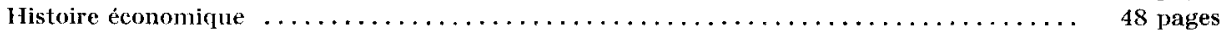

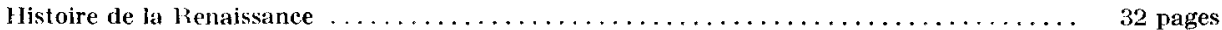

Le Secrétaire Général, Michel François. 


\section{CAHIERS DES ANNALES}

En vente à la LIBRAIRIE ARMAND COLIN, 103, boulevard Saint-Michel, 75005 PARIS Compte chèques postaux : Paris, No 21 335-25

2. Ch. MORAZE, Trois essais sur Histoire et Culture . . . . . . . . . . . . . 2,50 F

3. M. BLOCH, Apologie pour l'Histoire ou Métier d'historien, 6'édition . . . . . 9,40 F

6. L. E. HALKIN, Initiation à la critique historique, $4^{*}$ édition revue et corrigée . . 25,00 F

7. G. DEBIEN, En haut Poitou : défricheurs au travail (XV'-XVII' siècles) . . . . 3,90 F

8. A. TENENTI, La Vie et la Mort à travers l'art du XV' siècle . . . . . . . . . 5,50 F

11. G. DEBIEN, Etudes antillaises : au XVII' siècle . . . . . . . . . . . . . . 7,30 F

12. F. L'HUILLIER, La lutte ouvrière à la fín du Second Empire . . . . . . . . . 4,70 F

13. M. BLOCH, La France sous les derniers Capétiens (1223-1328) . . . . . . . . 6,30 F

14. M. DOMMANGET, Blanqui et I'opposition révolutionnaire à la fin du Second Empire . . . . . . . . . . . . . . . . . . . . . . . . 11,40 F

16. M. BLOCH, Seigneurie française et manoir anglais, $2^{\bullet}$ édition . . . . . . . . 11,40 F

17. M. DELAFOSSE et C. LAVEAU, Le commerce du sel de Brouage aux XVI' et XVIII siécles . . . . . . . . . . . . . . . . . . . . . . . . . . 9,40 F

18. A. DAUMARD et F. FURET, Structures et relations sociales à Paris au milieu du XVII" siècle . . . . . . . . . . . . . . . . . . . . . 8,20 F

20. H. ANTONIADIS-BIBICOU, Recherches sur les douanes à Byzance . . . . . 24,80 F

22. T. PLATTER, Autobiographie (texte tradult et présenté par Marie HELMER) . . , 11,40 F

24. M. MORINEAU, Jauges et méthodes de jauge anciennes et modernes . . . . 12,50 F

25. J.-P. ARON, Essai sur la sensibilité alimentaire à Paris au XIX siècle . • . 20,60 F

26. M. BLOCH, Souvenirs de guerre, 1914-1915 . . . . . . . . . . . . . . . . 9,20 F

27. Archéologie du village déserté, 2 volumes . . . . . . . . . . . . . . . 58,00 F

28. J.-J. HEMARDINQUER, Pour une histoire de l'alimentation . . . . . . . . . $39,00 \mathrm{~F}$

29. G. et M. VOVELLE, Vision de la mort et de l'au-delà en Provence . . . . . . 18,00 F

30. M. MORINEAU, Les faux-semblants d'un démarrage économique : agriculture et démographie en France au XVIII' siècle . . . . . . . . . . . . . . 45,00 F

31. H.-D. MANN, Lucien Febvre. La pensée vivante d'un historien. . . . . . . 30,00 F

32. J. MEUVRET, Études d'histoire économique. . . . . . . . . . . . . . . 35,00 F

33. A. ABBIATECl, F. BILl.ACOIS, Y. CASTAN, P. PETROVITCH, Y. BONGERT,

N. CASTAN, Crimes et criminalité en France (17'-18* siècles). . . . . . 22,00 F

34. M. MALOWIST, Croissance et régression en Europe (XIV'-XVII' siècles) . . . $4100 \mathrm{~F}$ 


\section{ÉCOLE PRATIQUE DES HAUTES ÉTUDES - VI SECTION SCIENCES ÉCONOMIQUES ET SOCIALES CAHIERS DES ANNALES, 34}

\section{MARIAN MALOWIST \\ CROISSANCE \\ ET RÉGRESSION EN EUROPE (XIV-XVII' SIÈCLES)}

Ce volume réunit un choix d'articles de l'historien polonais qui les avait publiés dans différentes revues au cours des vingt dernières années. II y étudie les rapports économiques de l'Europe de l'Est avec l'Occident, en particulier dans la zone de la mer Baltique, et les aspects sociaux de ces rapports. I/ cherche à expliquer pourquoi les pays de l'Europe du CentreEst, qui vivaient en pleine expansion économique au bas Moyen Age, retombèrent dans une période de longue stagnation, voire de régression, dès le début du XVIIe siècle.

227 pages, $41 \mathrm{~F}$ 